\title{
THE NEW PENICILLINS
}

\author{
E. B. Chain \\ Dept. of Biochemistry, Imperial College of Science and Technology, London, S.W.7.
}

Sir Charles, Ladies and Gentlemen,

It gives me much pleasure to perform the task of introducing this morning's session which is devoted to a discussion of some aspects of laboratory, in contradistinction to clinical, work on the new penicillins.

The penicillin molecule consists of a nucleus containing a characteristic fused thiazolidine$\beta$-lactam ring system, which is common to all penicillins, and a side chain acid linked in peptide linkage to the amino group of the nucleus which differs in the different penicillins. The side chain acid of the most commonly used benzylpenicillins is phenylacetic acid. In the fermentation process used for the commercial production of penicillin the side chain acid is added to the fermentation medium and incorporated by the mould into the penicillin molecule. It has been known for some time that it is possible to make penicillins with different side chains by adding different side chain precursor acids to the fermentation medium, but the mould will incorporate only derivatives of acetic acid. This sets a serious limit to the biosynthetic method of obtaining penicillins modified in their side chain. Only one of the biosynthetically produced penicillins has found widespread clinical application; it has the phenoxy-acetic acid as side chain, is acid stable and therefore can be given orally.

Important progress was made in the production of penicillins with modified side chains when it was found in 1956 by members of the research staff of the Beecham Research Laboratories that the nucleus of the penicillin molecule cumulated in penicillin fermentation media when the fermentations were carried out in the absence of a side chain precursor. This discovery made it possible to produce, by acylation of the free amino group of this substance, a practically unlimited number of new penicillins with every conceivable acid, not just derivatives of acetic acid, as side chains. However, the purification of 6-aminopenicillanic acid from the culture medium was a laborious, time consuming and costly procedure. This situation changed completely and 6-aminopenicillanic acid became as readily available as the commercial penicillins themselves, when enzymes of amidase nature were discovered in microorganisms which were capable of hydrolysing the side chain acid of commercial penicillins. Of special importance in this respect was the discovery of amidases in organisms of the Esch. coli type capable of removing the phenylacetic acid side chain from the cheap benzylpenicillin, and these enzymes are now widely used industrially for the commercial production of 6-aminopenicillanic acid.

As was to be expected, a very large number of new penicillins were made by acylation of this compound, and many of these have found clinical application. The question of the assessment of the clinical value of these new therapeutic agents therefore poses an acute problem.

In the evaluation of the clinical value of the new penicillins laboratory methods play an important, even an essential role, yet is must be realised that however perfect, ingenious and complete, they have their limitations.

The three laboratory methods mainly used for the evaluation of new penicillins are pharmacological, chemical and microbiological.

The necessity for careful pharmacological studies of any new compounds to be used therapeutically in man is so obvious that it needs no further comment, and this applies also to a class of compounds possessing generally so low a toxicity as do the penicillins. It is obviously essential to ascertain that they have no acute or chronic side effects in animals before they are extensively used on man. It is well to remember, however, that the pharmacological tests on penicillins have the same limitations as have those on other classes of compounds: the absence of toxicity in any of the known animal tests cannot be taken as an absolute guarantee that some new hitherto unknown toxic effect may not unexpectedly appear during clinical use. Vice versa, a very strong toxic effect of a substance in one animal species, such as for instance the very high toxicity of penicillin for the guinea pig (a few milligrams of penicillin are lethal to this species) does not mean that such compound is equally toxic for man and, as in the case of penicillin, does not preclude it from extensive clinical use. 
Chemical methods are used in the evaluation of new penicillins for ascertaining their stability in acid media and towards the enzyme penicillinase which is produced by many bacteria and has the property of opening the $\beta$-lactum ring of the penicillin molecule thus rendering it inactive against bacteria producing this enzyme. Many of the new penicillins were found to be acid stable and thus a whole series of new oral penicillins has become available.

Of far greater importance, however, was the discovery of penicillins stable against the action of staphylococcal penicillinase. These penicillinase resistant penicillins made possible the successful chemotherapeutic treatment of staphylococcal infection caused by penicillinase producing and therefore benzylpenicillin resistant staphylococci-this constitutes a notable advance in antibacterial chemotherapy.

Microbiological methods are used for determining the antibacterial powers of the new penicillins, for determining blood levels and for studying the capability of bacterial strains to develop resistance. The results will differ according to the assay method used. The serial dilution method will give antibacterial end points independent of the molecular weight of the compound to be tested whereas diffusion methods will give lower end points when the compound to be tested has a high molecular weight. Diffusion methods will also give lower end points if the antibacterial action of the compound is markedly influenced by the number of microorganisms against which it has to act. Which of the two methods reflects better the conditions in vivo? If the antibacterial compound has to act under conditions where diffusion presents no problems, such as for instance in the blood stream. and where the number of microorganisms against which it has to act is small. usually the serial dilution method may well be representative of the in vivo conditions. If, on the other hand, the compound has to act under conditions where diffusion is an important factor, the diffusion assay method would give a better picture of the actual in vivo condition in which the drug has to assert its function. Both the serial dilution method and the diffusion methods of antibacterial assay have therefore their own value and significance and complement each other in assessing the clinical value of a new penicillin. Both assay methods are, of course, influenced by the composition of the culture mediums. In particular, many antibacterial substances, including penicillins, are bound to serum proteins and for this reason have a lower antibacteria? activity in their presence. The binding of penie cillin to serum proteins occurs with different degrees of strength-in some cases it is ver. loose and reversible, in others it is firnses and irreversible. It is clear that penicilling which are readily inactivated by serum pro흘 teins will not be efficacious chemotherapeutice agents, but at the present state of knowledge, i is difficult to evaluate quantitatively the figures for reversible serum binding of the different new penicillins in the assessment of their clinica? efficiency, and the whole problem of serum $\vec{\omega}$ binding is at present the object of active investio gations in several laboratories.

Microbiological assay methods are widel $\stackrel{3}{\overrightarrow{2}}$. used for determining the blood level of peni- ${ }^{\circ}$ cillins after oral and parenteral administrationics Blood levels represent a balance between the absorption of the drug, the velocity of its? diffusion into the tissues and the rate of itso excretion. It is generally assumed that clinicaP efficiency and high blood levels of antibacteria $\square$ substances go together, but there are exceptionso to this rule.

Altogether, I believe that one must be veryo cautious in drawing conclusions from in vitro tests as to the behaviour of the drug undere clinical conditions. There are many factors affecting the clinical efficiency of drugs whistio we do not entirely understand. As our knowledge of these factors advances, our approach will become less empirical and more rational. At present we must, reluctantly perhaps, con- $\frac{\mathbb{D}}{\circ}$ clude that the only really valid tests for their clinical efficiency is their clinical performance, $\frac{\vec{z}}{3}$ and there is no short-cut to clinical trials.

The mode of action of the penicillins is a subiect which at present has no bearing on the evaluation of new penicillins though it may very 3 well have one in the future. It has, however, led: to most interesting advances in our knowledge 3 . of the structure of the bacterial cell wall. Dr. Park was the first to demonstrate that the penicillins act by interfering with the synthesis of 0 components of the bacterial cell wall; this explains the specificity of the antibacterialo action of the penicillins and their very low toxicity to animal tissues which do not contain such $\widetilde{\sigma}$ material.

It will be very interesting to hear a report from Dr. Park on the latest developments in this field and I have much pleasure to call on him to give the first lecture of this morning's session $\frac{C}{\Phi}$ on the mode of action of penicillin and the ? possible significance for therapy. 